\title{
Traduire
}

Une autre perspective sur r tr traduction

Revue française de la traduction

$236 \mid 2017$

Traducteurs en blouse blanche

\section{René Char en arabe}

Ahmed Mahmoud Khalifa

\section{(2) OpenEdition}

Journals

Édition électronique

URL : http://journals.openedition.org/traduire/925

DOI : 10.4000/traduire.925

ISSN : 2272-9992

Éditeur

Société française des traducteurs

Édition imprimée

Date de publication : 1 juin 2017

Pagination : $57-70$

ISSN : 0395-773X

\section{Référence électronique}

Ahmed Mahmoud Khalifa, «René Char en arabe », Traduire [En ligne], 236 | 2017, mis en ligne le 01 juin 2019, consulté le 10 décembre 2020. URL : http://journals.openedition.org/traduire/925 ; DOI : https:// doi.org/10.4000/traduire.925 


\section{René Char en arabe}

\section{Ahmed Mahmoud Khalifa}

\section{Introduction}

Imaginons que quelqu'un veuille traduire le poème de René Char en arabe. Dans cette langue, il n'aura même pas la ressource de traduire une ambiguité du texte français par une ambiguïté équivalente en arabe : celle-ci est grammaticalement impossible... (Mounin, 1981, p. 120)

Certes, mais traduire l'herbe, l'orage, l'insecte, le serpent, le requin et la mouette en arabe est-il tout autant admissible sur le plan poétique ? Le fait que Mounin ait jugé "impossible ", il y a plus de 30 ans, de traduire René Char en arabe est incontestable, surtout sur le plan de la linguistique. En effet, la linguistique a toujours dit « non " à la traduction(1). Mais l'existence des traductions arabes de l'œuvre de René Char suscite à son tour d'autres questions : qui a "pu " le traduire ? Comment a-t-il " pu " le traduire ? Quand ? Et pourquoi ? Cet article propose une méthode consistant à analyser deux corpus : le corpus paratextuel des traductions, limité à un seul élément de l'épitexte (l'allographe officieux) et un seul élément du péritexte (la préface) ; et le corpus textuel qui est indubitablement restreint, générique et en majorité au format numérique(2). Ce dernier sera ensuite soumis à une analyse poétique de la forme et du fond en les comparant avec ceux du texte d'origine afin d'évaluer les pertes et les gains de l'opération traduisante.

(1) Voir Martinet (1970), p. 36-37.

(2) II s'agit d'abord des deux traductions publiées en format livre puis de celles publiées sur des sites web littéraires. Celles-ci ont probablement paru dans le passé, avec des tirages très limités, dans quelques revues littéraires très spécifiques. 


\section{1) René Char : un parcours contrarié, une présence en pointillés}

Peu de recueils de René Char (1907-1988) sont traduits en arabe. Ce poète français, dont le parcours littéraire est très contrarié(3), mais aussi très réussi(4), n'a que deux livres publiés sous son nom dans cette langue(5). Pourtant, outre ces deux livres imprimés, il existe bel et bien d'autres traductions de plusieurs poèmes qui ont précédé ou suivi les publications précitées. Des poèmes traduits publiés dans des revues littéraires(6), des journaux, sur des sites internet(7) ou lors de forums littéraires consacrés aux amateurs de littérature étrangère, mais qui marquent tous la présence en pointillés du poète dans le champ littéraire arabe. Cette présence, plus ou moins visible, est en quelque sorte le résultat du pouvoir symbolique exercé par le champ littéraire français sur le champ littéraire arabe. En effet, si la majorité du corpus des traductions arabes de René Char se concentre sur ses écrits entre 1938 et 1945, c'est parce qu'il s'agit des années où les contextes historique, politique et social ont largement contribué à imposer ce poète comme figure littéraire au centre de son champ littéraire. Après la guerre, Char devient un grand poète parce qu'il a été un grand résistant. Les écrits de Char après 1945 ont moins marqué en France parce que le contexte de la Libération avait disparu. Le monde arabe admet également cette réalité en négligeant presque tous les écrits d'après 1945. La Capitale des Lettres exerce donc encore son pouvoir, même sur les écritures les plus prestigieuses.

(3) Le talent poétique de René Char fut l'objet de plusieurs débats critiques souvent influencés par des intentions politiques. Le poète est passé du salut littéraire des années 1940 aux critiques sévères de la fin des années 1950. II a d'ailleurs été salué puis critiqué par la même personne dix ans plus tard. Les écrits de Georges Mounin en témoignent : Avez-vous lu Char ? (1947) et "situation présente de René Char " dans Les Temps modernes (1957). Le poète aura la reconnaissance absolue à la fin de sa vie.

(4) Un parcours littéraire commençant officiellement par Le Marteau sans maître (1934), l'un de ses premiers recueils, édité au nom de l'amitié par José Corti, et s'achevant par l'intégration des CEuvres complètes du poète dans le catalogue de la Pléiade chez Gallimard en 1983.

(5) La première traduction est celle de son recueil Les voisinages de Van Gogh (1985) par Waḍḍāh Sharārah, publiée chez Dār al-Jadīd en 1989 à Damas (Syrie). La deuxième traduction est une sélection de poèmes tirés de quatre recueils : La fontaine narrative, Le poème pulvérisé, Partage formel et Feuillets d'Hypnos. Cette traduction porte le nom de Mushātarah Shakliyyah (Partage formel), éditée chez Manshūrāt al-Mujamma‘ al-Thaqāfĩ à Abou Dhabi en 1995.

(6) Ainsi, quelques poèmes de René Char ont été traduits dans la revue Shi'r dans les années 1980. Je n'ai pas pu les consulter. Il est quand même fort possible que ces traductions soient postérieures à l'intégration de René Char dans la Pléiade de Gallimard.

(7) J'ai choisi 22 poèmes traduits par différents traducteurs et disponibles sur le site internet de la littérature arabe : http://adab.com. 
Quelques traductions de René Char vers l'anglais, l'allemand et le russe sont jugées réussies(8). Plusieurs raisons à cela : d'abord il s'agit de langues ayant une certaine parenté référentielle et culturelle avec la langue française ; ensuite, faites du vivant du poète, elles sont très proches temporellement de l'œuvre originale. Traduire René Char vers l'arabe supposait a contrario de prendre en considération quelques critères : écart linguistique entre le français et l'arabe, écart temporel, écart contextuel et enfin écart poétique. Celui-ci sera examiné en détail dans l'analyse des textes traduits.

\section{2) Le paratexte arabe de René Char : surinterprétation et mésinterprétation}

La conception théorique de Gérard Genette(9) classe le paratexte en deux grands types : l'épitexte et le péritexte. L'épitexte est considéré comme une première " fréquence " de réception dite " intelligente " de l'œuvre littéraire. II s'agit d'écrits critiques faits par un public très restreint, un réseau très limité et très spécialisé, mais qui va s'ouvrir, grâce à internet(10), à un espace social illimité et délocalisé. Char est avant tout un poète rarement édité en arabe et cela doit nous amener à supposer que le débat critique autour de son œuvre en arabe est purement intellectuel. Théoriquement, tous ces articles critiques sont appelés " allographe officieux " et sont divisés en " allographe traduit ", " allographe auto-traduit " et " allographe récepteur ".

(8) Quelques poèmes de Char ont été traduits en allemand par Paul Celan en 1950. II s'agit de l'une des traductions les plus exactes de l'œuvre charienne jusqu'à nos jours, parce que le traducteur transmettait les aphorismes et les maximes, propres au style du poète, en se tenant au plus près du sens premier des mots et en donnant toujours la préférence à des emprunts au français. Ainsi, il traduisait " occupants " par "Okkupanten ", " ogres " par "Oger " ou " acteur " par "Akteur ". D'autres traductions allemandes faites par Johannes Hübner et Lothar Klüner ou Peter Handke, amis proches du poète, ont eu moins de succès parce que Char avait été amené à donner plus de précisions et de nuances en expliquant ou résumant brièvement ses poèmes (Greilsamer, 517). La traduction anglaise de Jackson Mathews en 1952 a été une véritable peine pour le poète puisque le traducteur avait demandé plus d'explications, de décortication des vers, de sondages des mots. La traduction russe faite par Vadim Kosovoï en 1972 a été saluée par René Char lui-même qui entretenait des relations amicales avec son traducteur-poète (Ibid., 515). Néanmoins, dans tous les cas, aucune de ses traductions n'a réussi à captiver un public de lecteurs aussi large que celui de l'après Libération en France.

(9) Voir Genette (1987).

(10) La plupart des articles que je mentionnerai ici sont publiés sur internet. Rares, voire absents, sont les articles publiés sur la poésie de René Char dans les revues littéraires du monde arabe. La tradition dans la traduction de la poésie française moderne vers l'arabe est de présenter le poète avec l'intégration de la traduction de quelques-uns de ses poèmes. Ainsi, le présentateur est souvent le traducteur. Ce qui éloigne ce type d'articles du corpus que je tenterai d'analyser ici. 
L'allographe traduit sur René Char se limite à deux articles dont je cite seulement un exemple(11) : celui concernant la publication du livre René Char en ses poèmes (1990) de Paul Veyne, dont le titre oriente déjà la réception : "L'un des plus grands historiens de la France se transforme en élève face à un poète " (Șāliḥ). Dans cet article, Char est un poète "surréaliste " et " radical "à la fois, un homme de lettres " engagé " mais qui déteste "les gangs des lettres ". L'allographe auto-traduit n'échappe pas à la même contradiction, même s'il s'agit d'auteurs francophones ayant lu Char en français avant d'écrire leurs articles en arabe. Cette élite bilingue, dont la fonction est de rapporter l'essentiel sur le poète dans l'objectif de l'inscrire dans son champ littéraire, dessine René Char d'une manière très différente. Ainsi nous pouvons lire, à titre d'exemple :

À travers sa complexité, à travers sa merveillosité, René Char est devenu, petit à petit, le poète à mentionner sans avoir besoin de le comprendre, sans avoir même besoin de le lire. II faut le momifier dans son hermétisme sans se rendre compte de sa facilité linguistique [...] Même si nous ne comprenons pas, nous ne pouvons pas cacher notre admiration pour un vers comme celui-ci (Quand tu es ivre du chagrin, tu n'as que le chagrin de cristal(12)). (Habash)

Ou encore :

René Char, cet homme soufi qui a merveilleusement chanté la vie dans ses plus beaux états. II a aussi chanté ce qui est derrière la vie, ce qui est avant elle, ce qui est au-dessus et au-dessous d'elle. La poésie de René Char est la chanson intarissable de l'homme. (Al-Sukhayrī).(13)

Et si l'allographe traduit et l'allographe auto-traduit annoncent leur impuissance face à la poésie de René Char, l'allographe récepteur, celui des lecteurs des traductions arabes, est souvent centré sur le poète lui-même. Pour son public de lecteurs arabes, Char est encore aujourd'hui le poète "résistant " qui a pu mener une conversation "fructueuse " avec un philosophe " nazi ", Heidegger (Al-Mișbāhīi). Ou encore le poète à qui le président de la République François Mitterrand rend visite pour oublier les soucis de la gouvernance, dans une sorte de pèlerinage spirituel vers la source de la poésie (Anonyme 1). La poésie de René Char, peu présente dans l'allographe récepteur arabe, souffre à son tour d'une description discordante, voire contrariée. Char est par exemple un poète " autiste " formé par le romantisme allemand mais qui était, en même temps, très réactif par rapport aux événements de son champ littéraire et de son champ politique (Al-Hāyik). Ou encore un poète " moraliste " vénérant " la beauté " mais qui est également un " surréaliste " recourant à la violence armée ('Awwād).

(11) II s'agit d'un article qui n'est en effet qu'une traduction d'une réception de Char dans son champ d'origine, c'està-dire une traduction d'un article écrit en français sur René Char, ce qui écarte toute intervention de la traduction de l'œuvre elle-même dans la réception.

(12) L'auteur voulait dire "C'est quand tu es ivre de chagrin que tu n'as plus du chagrin que le cristal ", in Char (1962), p. 103.

(13) Les deux citations mentionnées sont traduites par l'auteur du présent article. 
"l est également le poète qui s'est nourri de l'« illusion ", qui s'est trouvé une place privilégiée entre folie et génie et qui a su réaliser les "équations " poétiques les plus difficiles : clarté et obscurité, douceur et acerbité, attention et brutalité, truisme et étrangeté, pureté et saleté (Anonyme 2). Ou finalement le poète qui refuse d'être " en accord avec le rythme de la pratique poétique " parce qu'il veut " inventer sa propre pratique ", mais qui, en même temps, écrivait dans toutes les formes poétiques reconnues, poème libre, en prose, rythmé, rimé, etc. (Anonyme 3).

En ce qui concerne le péritexte arabe de l'œuvre de René Char, il se limite dans le présent article à une seule préface faite par l'un de ses traducteurs. II s'agit de Shākir La'ībī, qui a préféré commencer sa préface intitulée "Est-il possible de traduire René Char en arabe ? " par la phrase suivante : "La position de René Char par rapport à la poésie française moderne se résume dans sa réussite à créer une autre langue poétique dotée de la pensée elle-même " (La'î̄ī, p. 3). Le traducteur a ensuite signalé que la poésie de Char est " une pratique spéciale de la langue " (Id.) influencée à la fois par les écritures de Rimbaud, Hegel, Baudelaire et Heidegger. Mais, à l'inverse, il insiste sur le fait que la poésie de Char est une révolution contre la poésie et la philosophie elles-mêmes (Id.). Le poème de René Char, pour son traducteur préfacier, contient, à la différence d'autres poésies, des "dimensions " linguistiques et existentialistes "lointaines " (Id.). Chez le poète, rétorque le traducteur, "être " et " existence " se répètent souvent pour insister sur la nature " terrestre " de sa poésie (p. 4). Pour Char, " prose " est " poésie ", " poésie " est " philosophie ", donc " prose " est " philosophie ", confirme son introducteur dans la culture arabe qui déclare que, pour le lecteur arabe ou non-arabe, la poésie de Char est très difficile à comprendre parce qu'elle est " encombrée " de réflexions. Tous ces propos confirmés par le traducteur de Char vers l'arabe témoignent d'une certaine compréhension de la difficulté de la traduction poétique. Ses questions et ses déclarations en début de préface préparent le lecteur à affronter une traduction qui pourrait être " difficile " mais cela est dû au fait qu'elle est "réfléchie ", " soutenue " et " exacte ", même si elle n'est pas littérale : une traduction "poétiquement " exacte. Cependant, le reste de la préface, 25 pages environ, montre également qu'aucune déclaration faite auparavant n'a été confirmée. Aucune question n'a trouvé de réponse. Aucune proposition n'a été appliquée. Le reste de la préface est en effet " un dialogue violent " mené par le traducteur vis-à-vis des précédentes traductions de Char.

Le paratexte arabe existant sur René Char, même celui fait par ses traducteurs, annonce donc une réalité intéressante : rares sont les articles, sur le poète ou sur sa poésie, écrits à partir d'une lecture de la traduction des textes. Char est un poète français lu en français, même dans le monde arabe. Son public arabophone est également francophone : une élite bilingue, cultivée, qui a ses propres conceptions artistiques, directement influencées par celles du champ littéraire français. Le paratexte arabe de René Char n'est pas indépendant, il est influencé par le paratexte français. II s'agit d'une réception de la réception d'origine. Même la présentation de Char par son traducteur repose sur l'interrogation de la philosophie, de l'image, de la syntaxe 
difficile de la poésie charienne, en limitant la traduction à une interrogation sur les équivalents. Aucune question donc sur la philosophie ou l'image que pourrait engendrer la traduction ellemême. Aucune question sur le gain dit " poétique " de la traduction.

\section{3) Le texte arabe de René Char : à la recherche de la poétique}

En général, la poétique implique l'interrogation du fond, de la forme, du rythme, de l'image, etc. Mais la poétique de la traduction implique une série de questions supplémentaires concernant l'ajout, l'effacement, le choix des mots et même le choix des poèmes à traduire.

\section{a. La poétique est dans l'ajout, dans l'effacement, dans le choix des mots}

L'ajout, l'effacement et le vocable retenus par certains traducteurs travaillant sur l'œuvre de René Char ont complètement changé son sens, voire changé son identité et sa poétique. Mais, rares sont les poésies traduites qui gardent l'identité d'origine. La traduction neutralise tout et ne propose pas d'identité "équivalente ". L'identité de la poésie traduite est qu'elle est "traduction".

Dans les cas où la poétique du texte source repose sur la proposition concise, sur la puissance et l'intensité des mots pour distinguer une écriture dite "nerveuse ", nous trouvons que la traduction propose une autre "poétique cible " reposant, entre autres, sur l'étoffement, la répétition et la redondance pour marquer une écriture "calme ", voire de nature " testamentaire ". L'exemple le plus adéquat est celui de la traduction du poème "Le visage nuptial " par la traductrice tunisienne Āsiyah al-Sukhayrī, qui, par souci de "poéticité ", avait traduit " J'aime(14) " par " J'adore, je suis adorateur, je suis l'adorateur(15) " ou ailleurs la phrase "Tu as été créé pour des moments peu communs(16) " par "Tu as été créé pour des moments non dominants, tu as été créé pour tes propres moments(17) ». De cet allongement(18), qui s'est fait

(14) Char (1962), p. 32.

(15) (أنا أعشق... أنا عاثق... Al-Sukhayrī, Al-Wagh al-'Ursī.

(16) Char, Le Marteau sans maître, (1962), p. 32.

(17) Al-Sukhayrī, Al-Mitraqah Bi-Dūn Mu'allim.

(18) Le rapport poétique que cette traductrice entretient avec le texte de René Char est un vrai travail que je qualifie d'ı idéologique ". Le poème chez René Char est une vraie révolution contre la domination esthétisante de son époque. Le travail de la traductrice vers l'arabe se démarque par la pratique subjective, non seulement des ajouts, mais aussi des suppressions pour correspondre à une idée toute faite de la langue et de la poésie dans l'objectif de donner une élégance littéraire à sa traduction. Celle-ci, de ce point de vue, est concrètement une reproduction idéologique d'un texte déjà considéré comme production anti-idéologique (poème en prose par exemple). 
au détriment de "la masse brute du texte source(19) ", le texte arabe a gagné plus de " parlance " et de " signifiance "(20). II a gagné un autre aspect poétique remplaçant celui perdu par la traduction, un aspect poétique fondé par le traducteur.

La poétique réside également dans l'effacement. L'exemple de la traduction du poème "Afin qu'il n'y soit rien changé(21) " par le traducteur libanais Būl Shā'ūl montre bien qu'il s'agit d'une certaine volonté d'effacer. Le poème composé de neuf maximes, ou " notes " selon l'expression de René Char, se trouve réduit dans sa version arabe à seulement quatre fragments $(1,2,3,7)$. Le même poème sera retraduit plus tard par le même traducteur mais toujours réduit à quatre fragments $(1,2,3,4)$. Un autre exemple flagrant se trouve dans la traduction du poème " L'Extravagant(22) " par le traducteur libanais Hinrī Farīd Șa'b. Ce poème contenant trois cent seize mots dans sa version originale en contient seulement soixante-dix dans sa version traduite. Extra-effacé(23), ce poème n'est en effet que la traduction des deux premières phrases et de la dernière phrase du poème. Char, le maître de l'aphorisme, se trouve alors encore plus "résumé " en arabe.

L'effacement fait donc partie des pratiques de la traduction qui influencent indirectement la poétique du texte, tandis que le choix des vocables est une pratique directe sur le texte. Un exemple en est "Le requin et la mouette(24) ", un poème traduit d'abord par Șa'b, puis retraduit par al-Janābī. La deuxième traduction incarne le travail idéologico-poétique qu'exercent certains traducteurs sur le texte traduit. Al-Janābī s'est efforcé de rattraper les erreurs dites " poétiques " du premier traducteur. Son action porte essentiellement sur le vocabulaire. Ainsi, il traduit " requin " par kawsag(25) (poisson-scie), qui est un mot peu connu voire d'usage très rare en arabe, ou encore le " Je " qui débute le poème - supprimé d'ailleurs par le premier traducteur - par hā anā (Me voilà), il change toute la formulation du poème en arabe puisqu'il attribue une "surpuissance " au poète qui, dans l'œuvre originale, ne faisait que marquer sa présence. Ainsi, la phrase charienne écrite en italiques " J'ai levé la loi, j'ai franchi la morale, j'ai maillé le cœur ", traduite initialement par " J'ai annulé la règle, j'ai dépassé la morale et j'ai fermé le cœur(26) ", se trouve transformée en " J'ai enlevé la loi, j'ai dépassé les mœurs, j'ai diffusé le cœur comme un filet(27) ". Le mot " filet " joue ainsi un rôle décisif dans la construc-

(19) Voir Berman (1999), p. 53.

(20) Id.

(21) Voir Char (1962), p. 26-28.

(22) Id. p. 189-190.

(23) Sa'b, Al-Khāriq.

(24) Voir Char (1962), p. 197-198.

(25) Al-Janābī, Al-Kawsag wal-Nawras.

(26) (ألغيت القاعدة وتجاوزت الخلقية وزردت القلب.), Sab, Al-Qirsh wal-Nawras.

(27) (أزلت القانون وتجاوزت الأخلاق ونشرت القلب شبكة.), Al-Janābī, Al-Kawsag wal-Nawras. 
tion d'une image poétique. Remarquons que, si l'obstacle réside plutôt dans l'expression « mailler le cœur "que les deux traductions se sont efforcées de transmettre en arabe, il existe une grande différence entre "fermer le cœur " et " diffuser le cœur ". La différence entre un poète qui s'enferme, se replie sur lui-même, et un poète qui s'ouvre au monde, qui se sert de son cœur pour piéger "la vérité cachée ", voilà ce qu'on appelle la " figure du poète ".

\section{b. La poétique est dans la voix, dans l'image}

Un des deux livres de René Char en arabe est la traduction de son recueil le plus célèbre, Feuillets d'Hypnos, composé de 237 énoncés courts et successifs sous forme de bribes, de fragments et d'aphorismes. C'est une écriture qui essaye de dire la guerre à travers l'acte de communication, où la communication est elle-même en crise. Pour illustrer cette complexité, il suffit de rappeler que l'état de guerre nécessite en général que "les ponts " entre les mots soient « coupés (28) ». Char précisait à propos de ses notes poétiques : " Elles furent écrites dans la tension, la colère, la peur, l'émulation, le dégoût, la ruse, le recueillement furtif, l'illusion de l'avenir, l'amitié, l'amour. C'est dire combien elles sont affectées par l'événement(29) ". Feuillets d'Hypnos est un recueil dont l'objectif n'est certes pas de produire un énoncé cohérent et signifiant.

\section{Ponctuation et typographie}

La voix du poète et les mouvements de la parole s'introduisent dans l'écriture par la ponctuation et la typographie. La ponctuation fait entendre la poétique. Quand le poète veut marquer les différents registres dans son poème, il recourt le plus souvent à la segmentation par l'introduction de pauses. Celles-ci sont indiquées par des signes de ponctuation comme la virgule, le point, le tiret, les parenthèses ou les guillemets(30). Les traducteurs ont souvent tendance à traduire la ponctuation telle qu'elle est dans le texte original, sans se poser de questions sur la problématique même de sa traduction. Dans la langue arabe, la ponctuation ne fait partie de la tradition de l'écrit que depuis une époque très récente. Et même si elle est fréquemment utilisée aujourd'hui, elle est toujours limitée à son sens premier : séparation entre les syntagmes, marque de pause, d'interrogation ou d'exclamation. Seul le traducteur Shākir La'īî̄ avait toujours tendance à interpréter la ponctuation de René Char, comme par exemple dans "L'intelligence avec l'ange, notre primordial souci " (Feuillet 16) qui est devenue en arabe "l'intelligence et l'ange c'est notre premier souci(31) " : ici, la virgule, qui désigne l'“ identité " dans le texte source, est traduite par le verbe être à la troisième personne du singulier, hūwa

(28) Voir Dessons (1991), p. 138.

(29) Id. p. 139.

(30) Les exemples utilisés dans ce point de l'article sont tirés d'une étude littéraire consacrée au recueil Feuillets d'Hypnos de René Char, Dessons (1991), p. 138-147.

(الذكاء والملاك هو همنا الأولي.) (31), La'ībī, p. 77. 
(c'est, il est). Dans une autre phrase comme " Je suis homme de berges - creusement et inflammation - ne pouvant l'être toujours de torrent " (Feuillet 147), la traduction propose " Je suis l'homme des berges - le creusement et l'inflammation - parce que je ne suis pas capable d'être toujours un torrent(32) ". On voit ici que le traducteur a saisi une certaine relation des tirets avec le reste de la phrase : mettre les tirets tels quels aidera-t-il le lecteur à comprendre qu'ils sont dans le texte pour exprimer la causalité (parce que), pour inciser un commentaire, pour décrocher la parole signalée, pour marquer à la fois l'énonciation et l'intonation dans le texte ? Ailleurs, quand Char utilise des guillemets, c'est pour indiquer un certain recul, un certain dégagement de la responsabilité comme dans "Du jour où je suis devenu "partisan", je n'ai plus été malheureux ni déçu " (Feuillet 117). La traduction propose "Depuis le jour où je suis devenu "partisan", je n'ai été ni malheureux ni déçu(33) ". Nous pouvons donc conclure que l'absence de signification poétique de la ponctuation dans la langue arabe pourrait "nuire à " la relation d'un traducteur aussi compétent que La'ībī, avec le texte de Char.

La typographie (blancs, lettres italiques, majuscules, etc.) joue souvent un rôle démarcatif de valeur rythmique et sémantique des mots dans Feuillets d'Hypnos. L'italique, par exemple, a pour fonction de réactualiser ou de " remonter " la signification d'un mot au profit d'un autre. Il est souvent remplacé par le caractère gras ou quelquefois, tout simplement ignoré par le traducteur. Ainsi, le sens de l'italique dans la phrase suivante était-il sûrement plus difficile à saisir : " Le génie de l'homme, qui pense avoir découvert les vérités formelles, accommode les vérités qui tuent en vérités qui autorisent à tuer " (Feuillet 37). Autoriser est une action de l'homme, mais pas n'importe quel homme, c'est l'homme dirigeant. Autoriser est en italique pour mettre l'accent sur le machiavélisme qui cherche toujours à se détacher de toute responsabilité meurtrière. Or, oubliant même de marquer l'équivalent en gras, le traducteur produit une phrase incompréhensible(34). Le sens que portent quelques autres mots en italique, ou en majuscules, est d'ailleurs très difficile à transmettre vers l'arabe en les mettant simplement en gras. Ainsi, l'insouciance vis-à-vis de la mort dans "Je ne suis plus capable de mourir..." (Feuillet 54); la circonstance de l'action et non l'action elle-même dans "Si l'homme ne fermait pas souverainement les yeux, il finirait par ne plus voir ce qui vaut d'être regardé " (Feuillet 59); le sous-entendu et l'implicite dans : "Que fera-t-on de nous, après ? (Feuillet 64) ; l'ironie dans " la perte de la vérité, l'oppression de cette ignominie dirigée qui s'intitule bien" (Feuillet 174); ou encore l'horreur soulignée par les majuscules dans "Un œil arraché, le thorax défoncé, l'innocent absorba cet enfer et leurs RIRES " (Feuillet 99). Nous comprenons donc pourquoi les traducteurs allemands et anglais avaient eu tant besoin de sonder chaque mot dans l'œuvre de Char.

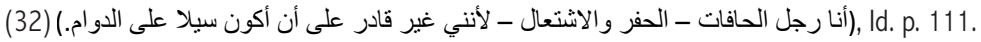

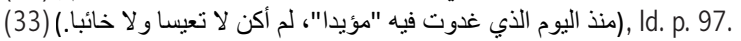

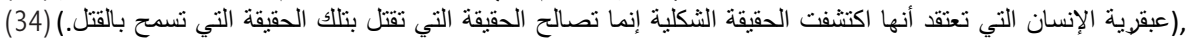
Id. p. 81. Ce qui veut dire en français " Le génie de l'homme qui croit avoir découvert la vérité formelle[,] ne fait que se réconcilier avec la vérité qui tue[,] avec celle-là même qui permet de tuer ". 


\section{L'image est un discours}

La métaphore rend, souvent, la poésie de René Char inaccessible ; il l'utilise pour échapper à la comparaison. Celle-ci pourrait l'obliger à proposer une relation analogique directe dans l'esprit de son lecteur or, il cherche plutôt à réaliser cette relation analogique au sein même du texte. Traduire l'image de Char est donc une opération délicate puisque tout le travail stylistique du poète risque de disparaître. Par exemple, les différents types de métaphores se trouvent détournés en arabe ou même déformés pour composer d'autres images. Ainsi, l'apposition "Les météores hirondelles " (Feuillet 175) devient " des météores d'hirondelles(35) ", l'attribut dans "le temps, c'est du chiendent " (Feuillet 137) est devenu "le temps est l'herbe(36) „. Sur un autre plan, la métaphore à complément de nom introduit par la préposition " de ", souvent utilisée par Char, est systématiquement mal traduite : " ma voix d'encre " (Feuillet 194) devient " la voix de mon encre(37) ", " sa maigreur d'ortie sèche " (Feuillet 178) est traduite par " la maigreur de son ortie sèche(38) ". Savoir pourquoi cette erreur de la traduction se répète me paraît une tâche très difficile puisque d'autres métaphores à complément de nom comme "l'écriture du soleil(39) " (Feuillet 181), "l'été doré de ses mains(40)" (Feuillet 176) sont pourtant exactement traduites. Quant aux métaphores à adjectif ou à verbe, elles sont souvent, mais avec quelques difficultés, bien transmises en arabe sans recours aux équivalences. Ainsi, des métaphores passent facilement en arabe comme "épaisseur triste(41) " (Feuillet 188), "bonheur bleuté(42) " (Feuillet 145), " la nausée écroule ma mâchoire(43) " (Feuillet 145), "les yeux seuls sont encore capables de pousser un cri(44) " (Feuillet 104), alors que d'autres ont du mal à être imaginées, telles que "présent crénelé(45) " (Feuillet 23) ou " l'aiguillon ne renonce pas à sa cuisson(46) " (Feuillet 39). D'ailleurs l'écriture de René Char établit souvent des liens entre des catégories logiques que la raison maintient séparées. Le rôle du traducteur, face à cette obscurité d'écriture, est sans doute primordial. Faut-il rendre clair l'obscur? Ainsi, " la chair folle du soleil " (Feuillet 37) devient " le corps fou du soleil(47) ", " les

(نياز ك من البلابل)(35), Id. p. 111.

(الزمان نجيل) (النابل), Id. p. 79. II faut également signaler que le traducteur a introduit une note en bas de page pour expliquer qu'il s'agit d'une sorte de mauvaise herbe et que cette mauvaise herbe pourrait désigner, dans un sens métaphorique, la difficulté et la confusion.

(37) (صوت حبري), Id. p. 115.

(38) (نحافة قر اصده الجاف), Id. p. 112.

(39) (كتابة الثمس), Id. p. 113.

(40) (كيف ييديه الذهبي), Id. p. 111.

(كثافة كئية) (لادية), Id. p. 114.

(42) (السعادة المزرقة), Id. p. 115.

(43) (يقوض الغثيان فكي), Id. p. 85.

(44) (ماز الت العيون لوحدها قادرة على إطلاق الصرخات), Id. p. 95.

(45) (حاضر مسنن), Id. p. 98.

(46) (المنخس لا يتخلى عن طبختهن), Id. p. 82.

(جسد الثمس المجنون), Id. p. 81. 
êtres exemplaires sont de vapeur et de vent " (Feuillet 228) devient « les êtres exemplaires sont la vapeur et le vent(48) ", "l'étanchéité de l'ennui " (Feuillet 41) se transforme en "l'étanchéité contre l'ennui(49) ॥. De ce fait, les oppositions travaillées par le poète entre l'animé et l'inanimé, l'humain et le non-humain, le concret et l'abstrait, se trouvent " rationnalisées ॥. Le choc que pourrait créer l'apposition dans "la chair du soleil " se trouve ainsi atténué, sinon rendu complètement logique dans "le corps du soleil ". "Les êtres " composés de "vapeur " et de "vent " deviennent la vapeur et le vent eux-mêmes. "L'étanchéité de l'ennui " qui est difficile à percer, se transforme en "étanchéité contre l'ennui ".

\section{c. La poétique est dans le rythme}

Le rythme est d'ailleurs l'élément le plus discuté dans les théories de la traduction, étant donné que la structure métrique et les musiques extérieure et intérieure du texte constituent en général son identité sonore. D'où l'exigence qu'un traducteur de poésie soit un poète doué d'un grand pouvoir mimétique sur le plan musical, qui lui permette de produire une traduction rythmée. Ainsi, "Allégeance(50) " est un poème en alexandrins, fondé sur des vocables et des résonances qui se répètent tout au long du texte. La répétition des mots, voire de strophes entières, n'existe malheureusement pas dans la traduction(51). La traductrice Āsiyah al-Sukhayrī, pour des raisons certainement idéologico-poétiques, a choisi de faire disparaître la résonance du texte original pour faire surgir d'autres résonances, plus difficiles à identifier. Sa transgression au niveau de la grammaire arabe a entraîné des faux-sens et des non-sens qui ne peuvent se justifier ni par la poétique ni par la logique de la langue cible, comme l'indique le poète arabe Adonis à propos de sa traduction de Bonnefoy : " Je préfère commettre des contresens scolaires plutôt que d'en commettre de poétiques.(52) "1. Ainsi, la poétique fondée sur le rythme, le mètre et l'image disparaît à cause d'un " Je " traduisant ayant choisi de défaire le texte original. " Un texte est à distance : on la montre, ou on la cache. Ni importer, ni exporter(53) ", disait Henri Meschonnic. La traductrice a donc choisi de montrer cette distance entre le texte de Char et sa traduction. En effet, le fait qu'un poème respectant le mètre et la rime soit organisé sous la forme d'un poème en prose porte un message du poète montrant qu'il peut y avoir de la poésie dans la prose. Le paragraphe ouvrant le poème se répète à la fin pour insister, entre autres, sur la musicalité du poème. Or, ce n'est pas le cas en arabe : le premier paragraphe n'est pas le quatrième. La traductrice a choisi de remplacer les répétitions par des synonymes, ce qui a eu pour effet de produire deux paragraphes différents.

(48)(الكائنات المثالية هي البخار و الريح.), Id. p. 124.

(49) (عازل للسأح) Id. p. 81

(50) Voir Char (1962), p. 228.

(51) Voir Al-Sukhayrī, Ikhlāș.

(52) Entretien réalisé par Usāmah Khayr-Allah, I'hebdomadaire Kull al-'Arab, Paris, 7 août 1987, in Hassan (2007), p. 208.

(53) Meschonnic (1973), p. 308. 


\section{Conclusion}

L'étude de ces traductions en arabe de quelques poèmes de René Char tend à montrer que la transmission est, hélas, incomplète. Cela s'explique d'abord par la faible compétence linguistique de certains traducteurs arabes et la forte idéologie poétique de ces traducteurs vis-à-vis de leur langue. Cette idéologie se manifeste non seulement dans leur stratégie de traduction (ajout, effacement, transformation, etc.) mais aussi dans leur stratégie d'écriture (choix des mots, reproduction du rythme et des images, etc.). Cet ensemble de pratiques exigeantes sur le poème de René Char, si particulier soit-il, a souvent déformé son sens, voire toujours sa nuance. Dans les rares cas où l'on trouve une traduction de belle formulation arabe correspondant au goût, à l'esthétique poétique de l'élite littéraire arabe, la confrontation avec le texte original pourrait facilement l'offenser. La poésie traduite de René Char témoigne d'une rareté de "belles infidèles " et d'une multiplicité d'" affreuses loyales ". Celles-ci, prosaïques et non rythmées, se trouvent encombrées d'images très complexes, voire incompréhensibles. Des images qui n'ont d'équivalent ni sur la terre ni dans le ciel arabes. Des symboles qui n'ont aucune signifiance. Des mots surdéterminés et dont le pouvoir référentiel se trouve naturalisé et amoindri. Les images bien transmises sont des images "universelles ". Le génie de René Char (l'implicite de son discours, les jeux de mots, les ambiguittés voulues) est sacrifié par ses traducteurs, naturellement centrés sur le sens immédiat des mots.

khalifa1980@azhar.edu.eg

Ahmed Mahmoud Khalifa est Maître de conférences au département de Langue et littératures françaises Faculté de langues et de traduction de l'université Al-Azhar en Égypte.

\section{Bibliographie}

\section{Corpus textuel}

LA'ĪBĪ Shākir, 1995, Mushāțarah Shakliyyah, Manshūrāt al-Mujamma' al-Thaqāfī, Abou Dhabi.

22 poèmes traduits par différents traducteurs disponibles sur le site internet de la littérature arabe (http://adab.com) sont mentionnés dans cet article :

AL-JANĀBĪ 'Abd al-Qādir, "Al-Kawsag wal-Nawras ", www.adab.com. http://adab.com/world/modules.php?name $=$ Sh3er\&doWhat $=$ shqas\&qid $=81850 \& r=c \_d e s \& r c=$ 14 (consulté le 17/3/2017).

AL-SUKHAYRĪ Āsiyah, " Al-Mițraqah Bi-Dūn Mu'allim », www.adab.com.

http://adab.com/world/modules.php?name=Sh3er\&doWhat=shqas\&qid=82026\&r=c_des\&rc= 12 (consulté le 17/3/2017). 
ID. "Al-Wagh al-'Ursī », www.adab.com

http://adab.com/world/modules.php?name $=$ Sh3er\&doWhat $=$ shqas\&qid $=82028 \& r=c \_d e s \& r c=$ 17 (consulté le 17/3/2017).

ID. " Ikhlās ", www.adab.com.

http://www.adab.com/world/modules.php?name=Sh3er\&doWhat $=$ shqas\&qid $=82024 \& \mathrm{r}=\& \mathrm{rc}=$ 11 (consulté le 17/3/2017).

SA'B Hinrī Farīd, "Al-Khāriq ", www.adab.com.

http://adab.com/world/modules.php?name=Sh3er\&doWhat=shqas\&qid=83086\&r=c_des\&rc= 19 (consulté le 17/3/2017).

ID. " Al-Qirsh wal-Nawras ", www.adab.com. s.d.

http://adab.com/world/modules.php?name=Sh3er\&doWhat=shqas\&qid=83085\&r=\&rc $=17$ (consulté le 17/3/2017).

\section{Corpus paratextuel}

'AWWĀD Mūsà, 2015, " al-Shā’ir wal-Fannān al-Firinsī Rīnīh Shār ", Ilà al-Amām, septembre, Baghdad.

AL-ḤĀYIK Nihād, 1988, « Rīnīh Shār, al-Shā'ir al-Mutawwaḥhid, Jawharī al-Kalimah », Āfāq 'Arabiyyah, Le Caire.

AL-JANĀBĪ 'Abd al-Qādir, "Rinīh Shār, Yab'ath al-Nashāṭ wa Yahra' Ilà al-Khātimah ", Jihat al-Shi'r, http://www.jehat.com/jehaat/ar/KheyanatThahabeya/poet/ (consulté le 17/3/2017).

AL-MIṢBĀḤ̄ Ḥassūnah, 2015, "Rīnīh Shār wa Hāydghar Tāḥta Shagarat al-Kistinā' ", al-lttihāā. AL-SUKHAYRī Āsiyah, "Rīn̄ih Shār, Sayyid al-Farah al-Mustaḥīl ", Arab World Book, s.d. http://www.arabworldbooks.com/ArabicLiterature/renechar.htm (consulté le 17/3/2017).

ANONYME 1, "Fī Dịyāfat al-Shā’ir ", al-Jarīdah, s.d.

http://www.aljarida.com/ext/articles/print/1461829933353496900/ (consulté le 17/3/2017).

ANONYME 2, "Būrtrīh Rīnīh Shār ", Al-Thawrah, 2382006.

http://thawra.sy/_print_veiw.asp?FileName=26367249020060822222641 (consulté le 17/3/2017).

ANONYME 3, "Rīnīh Shār wa 'Uzlat al-'Āṣifah ", Al-Bayyinah, s.d. http://al-bayyna.com/modules.php?name=News\&file=article\&sid=31521 (consulté le 17/3/2017).

HABASH Iskandar, "Qașā'id Fī al-Arkhabīl ", Jihat al-Shi'r, s.d.

http://www.jehat.com/Jehaat/ar/KheyanatThahabeya/poet/4-11-09.htm (consulté le 17/3/2017). 
ȘĀLIḤ Hāshim, " Aḥad Kibār Mū'arrikhī Faransā Yataḥawwal llà Tilmīdh Amām Shā'ir ", Al-Sharq al-Awsat, 2002.

http://archive.aawsat.com/details.asp?article $=118837$ \&issueno $=8664 \# . V g 1 \mathrm{hC}$ Tzgl1 (consulté le 17/3/2017).

\section{Études:}

BERMAN Antoine, 1991, La traduction et la lettre ou l'auberge du lointain, Paris, Seuil. CHAR René, 1962, Fureur et mystère, Paris, Gallimard.

DESSONS Gérard, 1991, Introduction à l'analyse du poème, Paris, Bordas.

GENETTE Gérard, 1987, Seuils, Paris, Seuil.

GREILSAMER Laurent, 2007, René Char, Paris, Éditions Perrin.

HASSAN Kadhim Jihad, 2007, La Part de l'étranger, la traduction de la poésie dans la culture arabe, Arles (France), Actes Sud.

MARTINET André, 1960, Éléments de linguistique générale, Paris, Armand Colin.

MESCHONNIC Henri, 1973, Pour la poétique : Essai, Paris, Gallimard.

MOUNIN Georges, 1981, "Envoûtement à la Renardière ", Langue française, volume 49, Paris, http://www.persee.fr/doc/lfr_0023-8368_1981_num_49_1_5086

VEYNE Paul, 1990, René Char en ses poèmes, Paris, Gallimard. 